\title{
Benzene's electron structure
}

\section{A new calculation shows that Kekule may have made a good model of benzene, but also fails to resolve the question whether valence bonds are better than molecular orbitals (or vice versa).}

Peter A. Schultz and Richard P. Messmer are at it again: that is the simplest thing to say of the paper the two theoretical chemists from the Department of Physics at the University of Pennsylvania at Philadelphia have now published on the electronic structure of benzene (Phys. Rev. Lett. 58, 2416; 1987). Under the title "Are there $\pi$ bonds in benzene?", the two authors say that the answer is probably negative, and that the best description of the benzene molecule is probably based on an oscillating ("resonating", vide Pauling) blend of the two obvious equivalent patterns of alternating double bonds originally proposed by Kekulé in 1872 .

Some recent parochial history of this tale is relevant. In October last year, Cooper, Gerratt and Raimondi from the Universities of Liverpool, Bristol and Milan, published (Nature 323, 699; 1986) an account of the electronic structure of benzene, now described by Schultz and Messmer as the "prototypic aromatic molecule". Cooper et al. concluded that the now-standard textbook description of the benzene molecule as a symmetrical and essentially static structure is probably incorrect. In particular, they concluded, the electrons supposed, in the usual textbook description, to form two hexagonally shaped rings above and below the otherwise planar structure of the molecule (the famous $\pi$ electrons) are probably more localized on the atomic nuclei than the standard view would allow.

A few weeks later, Schultz and Messmer published (Phys. Rev. Lett. 57, 2653; 1986) an equally interesting reappraisal of the electronic structure of a molecule, in that case $C_{2} F_{2}$. Again it seems that a valence-bond calculation of the structure of the molecule had shown that textbook expectations are not fulfilled. Acknowledging that the two pieces of work were demonstrably independent of each other, Nature nevertheless cheekily chided the second group of authors for having claimed their work to be the "first" of its kind (Nature 324, 599; 1986).

The latest development is more mystifying, given the history, for the latest paper refers neither to the original paper by Cooper et al. nor to the teasing commentary on the affair that appeared afterwards in Nature (but which Schultz and Messmer may well have judged beneath contempt). That, however, has an innocent explanation; surprisingly, it turns out that the latest paper from Schultz and Messmer, in the issue of Phys. Rev. Lett. for 8 June, had been submitted for publication on 2 September last year, six weeks before the original paper by Cooper et al. appeared in Nature. Why the paper has been hanging about all this time is a matter for the authors and the journal; otherwise the oldest paper in the issue of 8 June is dated early in February this year. But, in the circumstances, may not Cooper et al. have rated a "note added in proof", a common convention in pure physics journals having to survive the flood of preprints that threatens to overwhelm them?

The importance, such as it is, of this tale is as an illustration of how even the modern literature is not nearly as well-ordered as it might be. References to other people's work, conventionally the means by which authors acknowledge those (giants and otherwise) on whose shoulders they have stood, are also convenient cross-references between parallel developments. Perhaps some of the energy now being spent on making the literature electronic should be used, in passing almost, to solve that problem.

None of this is intended to distract, let alone detract, from what Schultz and Messmer (not to mention Cooper et al.) have done. The classical problem of benzene and other aromatic compounds is understanding why carbon atoms, which usually form four tetrahedrally arranged bonds with other atoms, should sometimes form trigonal bonds with added stability (as in benzene, where each carbon is bonded to one hydrogen and the two neighbouring carbon atoms in a hexagonal ring). Rational explanation came only in the late 1920 s, with the recognition that the spherically symmetrical $s$ and polar $p$ orbitals of the second quantum level are energetically degenerate and, with the neglect of inter-electron interactions, may be rearranged into a set of polar orbitals arranged trigonally in a place, with a symmetrical (strictly, an antisymmetrical) polar orbital, the famous $\pi$, at right angles to the plane.

The neatest feature of this argument is that it provides an easy way of calculating something. On the assumption that a benzene molecule is held in place by the within-the-plane bonds between carbon atoms (called $\sigma$ bonds), it is a fair question to ask what extra binding energy arises from the interaction between the ring of six dumbbell-shaped orbiting electrons at the vertices of a benzene ring. Naturally, the simplest calculations are grossly approximate; the simplest of all is to assume that electrons in the molecule as a whole are adequately described by linear combinations of the atomic $\pi$ orbitals (with complex coefficients to allow them to seem orthogonal to each other). But there are many other tricks that can be played, using the calculated wave functions of electrons in the force-field of a hexagonal charged structure, for example.

The other way of dealing with these problems is more intuitively appealing, based as it is on the arm-waving that passed for theoretical chemistry before quantum mechanics arrived. Given the valency of atoms and the geometrical propensities of their bonding patterns, it is relatively easy to construct all possible structures. The trick, easily translated into wave mechanics language, is then to suppose that the true state, in the sense of quantum mechanics, is a superposition of all possible states. It is like psychoanalysis, where one looks for the "baby" or the "destructive infant" part of a person.

In simple cases, the valence-bond approach allows calculations to be carried through, for the hydrogen molecule for example. For molecules as complicated as benzene, the calculations have only recently become realistically possible with the arrival of Cray computers and the like, but the alternative valence-bond structures that matter are those originally described by Kekulé.

The two calculations now described (Cooper et al. and Schultz and Messmer) are complementary. The first showed that electrons do tend to be more localized on the atoms at hexagonal vertices than the simple picture of entirely mobile $\pi$ electrons suggests. Schultz and Messmer have the more detailed pictures. Their particular contribution is their concept of what they call the "bent- $\Omega$ " bond.

But Schultz and Messmer raise one potentially contentious issue by saying that they believe valence-bond calculations to "have a significant advantage" over other approximations in retaining "an interpretability" with a direct relationship to the "almost universally accepted concepts of structural chemistry". But all these methods of calculation can only be approximations to the same truth and Mulliken's Chicago school, with its distinctive view of structural chemistry, would have hotly disputed the conclusion.

John Maddox 